\title{
Medicinal cannabis in the treatment of chronic pain
}

Luke A Henderson, Vicki Kotsirilos, Elizabeth A Cairns, Alister Ramachandran, Chris C Peck, lain S McGregor

\section{Background}

Chronic pain is a major health issue, adversely affecting millions of Australians and costing billions of dollars annually. Current pharmaceutical treatments may be limiting, and in some cases ineffective, while carrying substantial liabilities. Medicinal cannabis is an increasingly popular, albeit controversial, alternative.

\section{Objective}

The aim of this article is to briefly review the scientific evidence related to medicinal cannabis for the treatment of chronic pain and update physicians on relevant issues and optimal prescribing practices.

\section{Discussion}

To date, $>130,000$ medicinal cannabis approvals have been issued in Australia, mostly by general practitioners, with approximately $65 \%$ of these to treat chronic non-cancer pain. Available products deliver $\triangle^{9}$-tetrahydrocannabinol (THC) and/or cannabidiol (CBD). Despite robust supportive data from animal models, current clinical trial evidence for $\mathrm{THC}$ and $\mathrm{CBD}$ efficacy in chronic pain is incomplete. In their prescribing decisions, doctors must balance patient demand and curiosity with caution regarding potential risks and limited efficacy.
IN SOME INDIVIDUALS, ACUTE PAIN persists and develops into a chronic pain state, defined as daily pain lasting $>3$ months. ${ }^{1}$ Chronic pain is difficult to treat and can adversely affect quality of life and day-to-day function. Suicidal behaviour is 2-3 times higher in patients with chronic pain, and approximately $40 \%$ of forced early workforce retirements are due to chronic pain. One in five Australian adults are estimated to live with chronic pain, costing the community over $\$ 140$ billion per annum. ${ }^{1,2}$ Chronic pain is a frequent presentation in general practice and central to commonly treated conditions such as arthritis, fibromyalgia, cancer and diabetes.

Chronic pain can be divided into three mechanistic categories: nociceptive pain maintained by constant activation of pain receptors (nociceptors); neuropathic pain that results from lesions, disease or dysfunction of the nervous system with or without peripheral nerve changes; and nociplastic pain that results from nervous system sensitisation without clear evidence of tissue or nerve damage (Figure 1). ${ }^{3}$ Current interventions for chronic pain may vary somewhat according to the type of pain observed (eg opioids for nociceptive pain, and gabapentinoids or antidepressants for neuropathic pain). Current pharmacological interventions tend to be of limited efficacy, with fewer than $20 \%$ of individuals reporting pain relief of $50 \%$ or more. ${ }^{4}$ Even when pain relief is obtained, current medications can have significant side effects - including the potential for abuse/misuse - and are often unsuitable for long-term use. ${ }^{5}$

It is estimated that 600,000 Australians currently self-medicate with cannabis, ${ }^{6}$ with chronic pain a leading indication for such use. Most of this self-medication involves illicit cannabis, ${ }^{7}$ although a growing number of patients are now transitioning to prescribed medicinal cannabis products. Since late 2016, medicinal cannabis products can be legally prescribed by Australian doctors under the Special Access Scheme Category B (SAS-B) and the Authorised Prescriber Scheme of the Therapeutic Goods Administration (TGA). Most current prescriptions occur under the SAS-B scheme and involve doctors applying on behalf of individual patients to access a medicinal cannabis product. ${ }^{8-10}$ As of July 2021, >130,000 such approvals had been issued under SAS-B, with approximately $65 \%$ of these to treat chronic pain. After a very slow start in 2017-2019, approvals reached approximately 10,000 per month during early 2021 (Figure 2). Queensland has by far the highest per capita rate of SAS-B approvals, followed by Victoria and NSW (Table 1). Such 'approvals' are 
not necessarily clinical endorsements of medicinal cannabis; they simply indicate that TGA regulatory requirements have been met.

More than 190 different medicinal cannabis products can currently be accessed via TGA schemes, ${ }^{11}$ the most commonly prescribed being orally administered liquids (cannabis 'oils') with pharmaceutical grade cannabis plant material ('flower', 'flos' or 'bud') the second most common category (Figure 3 ). Other available products include capsules, sprays and wafers. Therapeutic actions of these medicinal cannabis products primarily arise from the cannabinoids $\Delta^{9}$-tetrahydrocannabinol (THC; the main intoxicating component of cannabis) and/or cannabidiol (CBD; a non-intoxicating component). Available products can be categorised as THC dominant or CBD dominant or can contain a mixture of THC and CBD (ratios of $\sim$ :1 and 1:10 are common). CBD-dominant products are in Schedule 4 of the Poisons Standard (>98\% of total cannabinoid content must be CBD), while products with a significant amount of THC are in Schedule 8 and may require additional state-level permissions to prescribe. A recent rescheduling decision by the TGA means that CBD-dominant oral products containing $<150 \mathrm{mg}$ $\mathrm{CBD}$ and $<1 \%$ THC may soon become available over the counter in Australian pharmacies.

The aim of this article is to examine the use of THC and CBD in the treatment of chronic pain and summarise the evidence related to efficacy, side effects and optimal prescribing practices. For further information about the use of medicinal cannabis in palliative care settings, these authors recommend the article by Herbert and Hardy. ${ }^{12}$
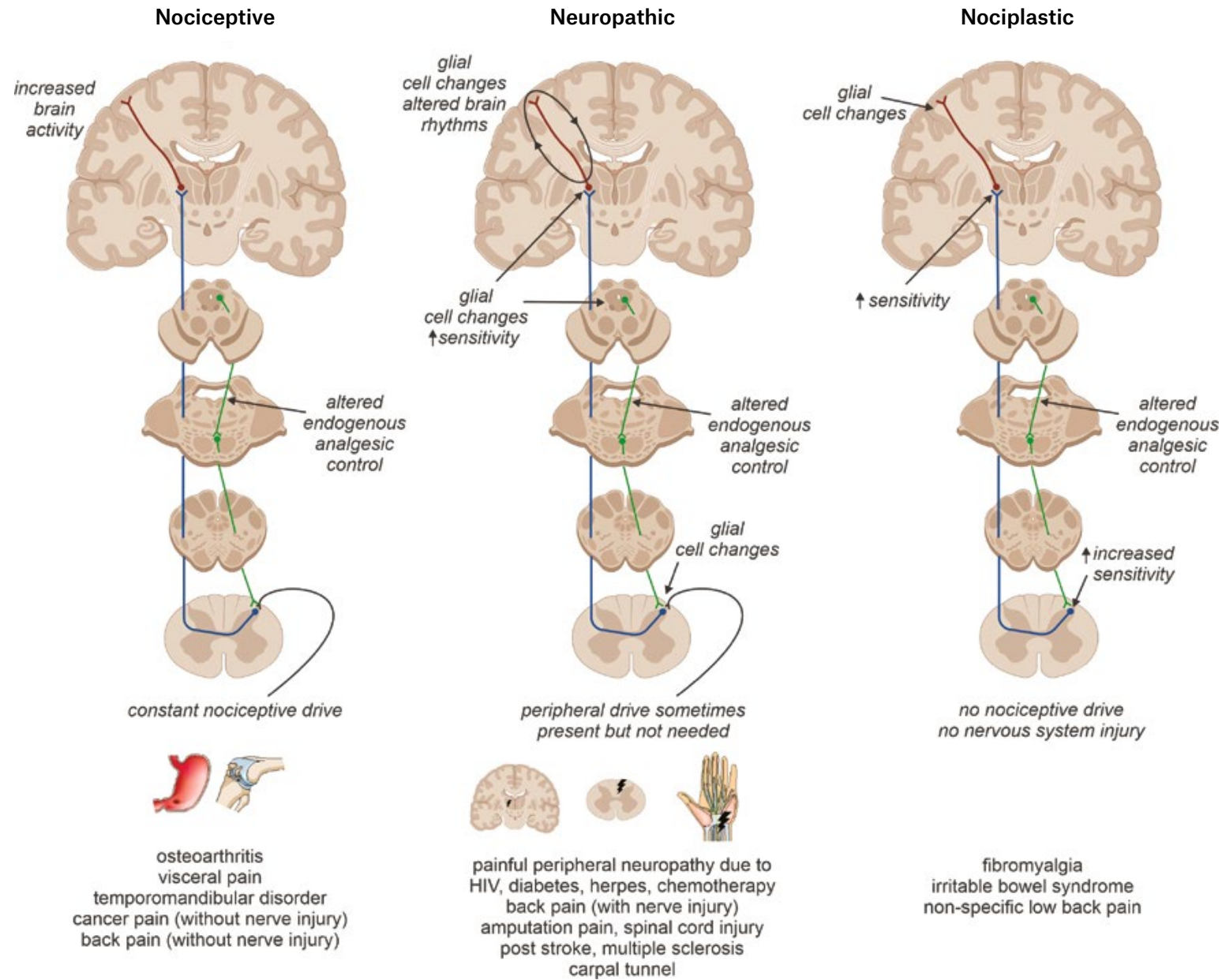

Figure 1. Chronic pain can be divided into three major categories: nociceptive, neuropathic and nociplastic. Chronic nociceptive pain is characterised by the presumed constant activation of peripheral nociceptors with limited changes in central nervous system function. Chronic neuropathic pain is characterised by damage or presumed damage to the nervous system; it is is characterised by significant changes within the central nervous system and can exist without peripheral input. Chronic nociplastic pain results from increased nervous system sensitisation with no clear evidence of actual or threatened tissue damage and without nerve injury.

$H I V$, human immunodeficiency virus 
The endocannabinoid system and pain

The analgesic effects of cannabinoids are well established in preclinical models of pain ${ }^{13}$ and arise primarily via interactions with the endocannabinoid system (ECS). The ECS is a ubiquitous system with multiple functions and comprises lipid signalling molecules called endocannabinoids (eg anandamide and

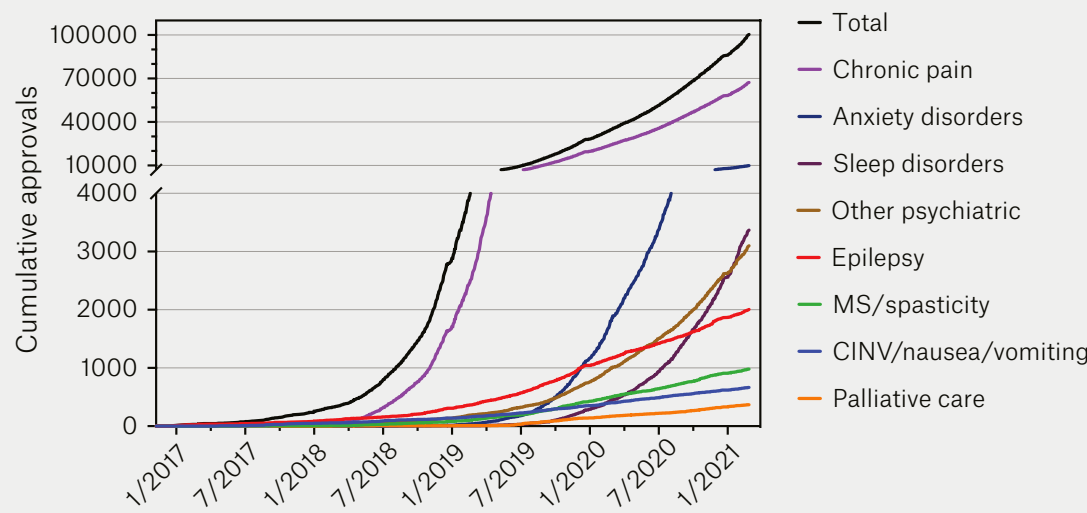

Figure 2. Recent medicinal cannabis approvals in Australia. Medicinal cannabis approvals in Australia from January 2017 - April 2021, sourced through a Freedom of Information request \#2275 to the Therapeutic Goods Administration. The approval rate for products to treat various chronic pain conditions is increasing dramatically. This increase highlights the growing incidence of chronic pain experienced by Australians, increase in patient demand for medicinal cannabis and the possible lack of alternative effective treatment options for chronic pain. There is an urgent need for clinical trials to assess the efficacy of medicinal cannabis for chronic pain relief.

CINV, chemotherapy-induced nausea and vomiting; MS, multiple sclerosis 2-arachydonyl glycerol [2-AG]) that bind to specific cannabinoid receptors ( $\mathrm{CB} 1$ and $\mathrm{CB} 2$ receptors) or other endocannabinoidsensitive receptors (eg GPR18, GPR55, GPR119, TRPA1, TRPV1). Specialised enzymes regulate the synthesis, transport and degradation of endocannabinoids to maintain overall endocannabinoid tone.

The critical role for the ECS in pain and inflammation can be seen from the effects of experimental drugs that inhibit fatty acid amide hydrolase (FAAH; an enzyme that breaks down anandamide and other endocannabinoids). Inhibition of FAAH leads to elevated systemic endocannabinoid concentrations and analgesic effects in animal models of pain. ${ }^{13}$ In humans, a recent case study describes an elderly woman with a genetic polymorphism that reduces FAAH expression leading to elevated endocannabinoid concentrations and pain insensitivity. ${ }^{14}$ Reduced FAAH function is also associated with reduced need for postoperative analgesia in women undergoing breast cancer surgery. ${ }^{15}$

\section{Phytocannabinoids}

The cannabis plant contains $>140$ cannabinoids (known as

Table 1. Total and chronic pain Special Access Scheme Category B (SAS-B) approvals (month of April 2021) by state/territory

\begin{tabular}{|c|c|c|c|c|c|}
\hline State/territory & All approvals & $\begin{array}{r}\text { Chronic pain } \\
\text { approvals }\end{array}$ & $\begin{array}{l}\text { Chronic pain } \\
\text { approvals (\%) }\end{array}$ & Population* & $\begin{array}{l}\text { Approvals } \\
\text { per capita }^{\dagger}\end{array}$ \\
\hline Queensland & 4,006 & 2,494 & 62.3 & $5,194,900$ & 77.11 \\
\hline New South Wales & 1,063 & 733 & 69.0 & $8,172,500$ & 13.01 \\
\hline South Australia & 51 & 41 & 80.4 & $1,770,800$ & 2.88 \\
\hline $\begin{array}{l}\text { Australian Capital } \\
\text { Territory }\end{array}$ & 20 & 12 & 60.0 & 431,500 & 4.63 \\
\hline Northern Territory & 7 & 2 & 28.6 & 246,600 & 2.84 \\
\hline
\end{tabular}

Data obtained via Freedom of Information request \#2370-02 to the Therapeutic Goods Administration, available at www.tga.gov.au/foi-disclosure-log

${ }^{*}$ Population data obtained from www.abs.gov.au/statistics/people/population/national-state-and-territory-population/dec-2020

${ }^{t}$ Per capita refers to number of approvals per 100,000 population

\#Includes other territories such as Jervis Bay Territory 
phytocannabinoids), with THC and CBD the most abundant and the best characterised. Pharmacologically, THC acts a partial agonist at $\mathrm{CB} 1$ receptors, this being the primary mechanism behind its distinctive psychoactive effects, as well as analgesia and sedation. A synthetic structural variant of THC called nabilone also acts as a CB1 receptor agonist and is available by prescription in some countries for the treatment of chronic pain (for studies of efficacy, refer to Turcotte et $\mathrm{al}^{16}{ }^{16}$ Bestard et $\mathrm{al}^{17}$ and Berlach et $\mathrm{al}^{18}$ ). THC also acts as a partial agonist at CB2 receptors, which are widely expressed on immune cells and have a key role in inflammatory and immune processes. The role of $\mathrm{CB} 2$ receptors in mediating $\mathrm{THC}$ effects and in analgesia is not entirely clear; effects of $\mathrm{CB} 2$ receptor agonists on inflammation-induced pain are better described than their effects on nerve injury-related pain. ${ }^{13}$ Notably, a CB2 receptor-preferring agonist, lenabasum, is currently in late-stage clinical trials for the treatment of various inflammatory autoimmune conditions and fibrosis. ${ }^{19}$

CBD, in contrast to THC, does not directly activate $\mathrm{CB} 1$ receptors, and this explains its absence of intoxicating effects. ${ }^{20} \mathrm{CBD}$ interacts, however, with a range of ECS-related receptors, and enzymes, to produce an array of anticonvulsant, anxiolytic, antipsychotic, anti-inflammatory and possible analgesic effects. ${ }^{21}$ While CBD appears analgesic in animal models, particularly those modelling neuropathic pain, ${ }^{13}$ there are minimal data related to analgesic effects in humans.

\section{Evidence for the efficacy of cannabinoids in chronic pain}

\section{Overview of evidence}

The evidence base for the efficacy of medicinal cannabis in treating chronic pain is complex and contentious. Numerous systematic reviews and meta-analyses have been conducted ${ }^{22,23}$ reaching both positive and negative conclusions. Although somewhat dated, a concise and useful review of outcomes is provided by the TGA's Clinical guidance for the use of medicinal cannabis in the treatment of chronic non-cancer pain (December 2017). ${ }^{24}$ This analysis concluded that medicinal cannabis products were superior to placebo in producing a $30 \%$ reduction in pain scores and a $50 \%$ reduction in pain intensity ratings. However, the overall quality of evidence related to efficacy was low. A related review, published by Australian researchers in

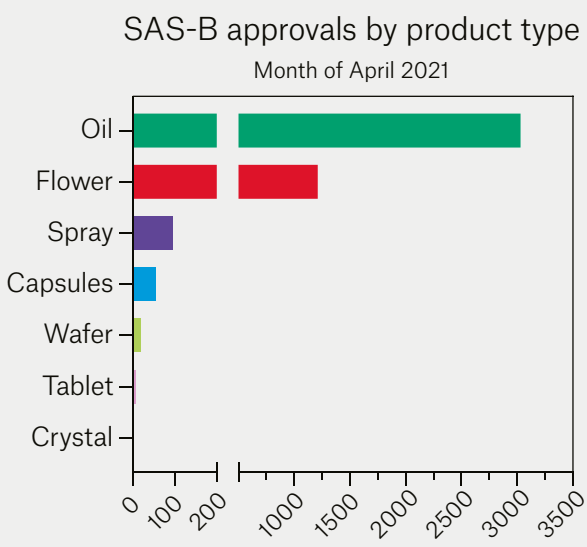

Figure 3. Types of medicinal cannabis products prescribed for chronic pain under Special Access Scheme Category B (SAS-B) in the month of April 2021. Data obtained through Freedom of Information request \#2370 to the Therapeutic Goods Administration. Orally administered oils are the most frequently prescribed products, followed by cannabis plant material ('flower').

2018, concluded that 'evidence for the effectiveness of cannabinoids in chronic non-cancer pain is limited' ${ }^{25}$ A more recent 'review of reviews' ${ }^{22}$ concluded that the 57 systematic reviews of the literature over the past 20 years were 'lacking in quality and cannot provide a basis for (clinical) decision making'. The array of studies reviewed in these systematic reviews involved a heterogeneous mix of cannabinoids, routes of administration, doses, pain conditions treated and outcome measures, with studies also differing on whether cannabinoids were used alone or adjunctively with other medications. It is striking that very few, if any, high-quality clinical trials have assessed the effects of the most commonly prescribed SAS-B products (ie orally administered cannabis oils) on chronic pain. Some key studies and outcomes are summarised in this article.

\section{Inhaled cannabis}

The most traditional route of cannabis consumption is inhalation of burned plant material, typically via bongs or joints. These modes are still widely used in the Australian community for self-medication with illicit cannabis, ${ }^{7}$ and herbal cannabis is now a popular prescription product that is inhaled via vaporisation (rather than smoking). Plant material currently accounts for nearly $30 \%$ of current SAS-B approvals (Figure 3), ${ }^{11}$ and a range of vaporisers have been approved by the TGA as medical devices for this purpose. ${ }^{26}$ The evidence relating to inhaled cannabis for chronic pain is varied. An early review of five clinical trials reported a $>30 \%$ pain reduction in conditions such as diabetic and human immunodeficiency virusrelated neuropathies. ${ }^{27}$ Other positive outcomes have been reported in trials of neuropathic pain. ${ }^{28-30} \mathrm{~A}$ large observational study involving thousands of Israeli patients documented reduced cancerrelated pain and improved quality of life in patients using smoked cannabis across periods of several months. ${ }^{31}$

\section{Nabiximols (THC/CBD oromucosal spray)}

Nabiximols is an oromucosal spray containing a $1: 1$ ratio of $\mathrm{THC} / \mathrm{CBD}$ 
that is currently listed on the Australian Register of Therapeutic Goods (ARTG). The main indication of nabiximols is spasticity in multiple sclerosis (MS), where it has well-demonstrated efficacy. ${ }^{32}$ Results relating to pain have been more mixed, ${ }^{23}$ including a marginal outcome in a large trial of nabiximols in cancer pain. ${ }^{33}$ Trials of nabiximols for pain associated with spinal cord injury, ${ }^{34}$ diabetes ${ }^{35}$ and chemotherapy ${ }^{36}$ have reached negative results; however, positive effects have been obtained in patients with brachial plexus avulsion ${ }^{37}$ and a mixed peripheral neuropathic pain. ${ }^{38}$ Analysis of a large German registry $(n=800$ patients) indicated that $70 \%$ of patients reported a $>50 \%$ improvement in pain after 12 weeks, with additional improvements in stress, depression, anxiety and overall wellbeing. ${ }^{39}$ Overall symptom relief/ improvement scores favoured neuropathic pain over nociceptive pain.

\section{Dronabinol (oral THC)}

Dronabinol is synthetic THC in capsule form that is available on prescription in some countries, although not in Australia. Dronabinol has shown positive effects in patients with MS-related neuropathy ${ }^{40,41}$ but failed in a study of neuropathic pain related to spinal cord injury. ${ }^{42}$

\section{Cannabidiol}

There have been very few clinical trials exploring the analgesic effects of CBD in humans. While CBD is a component in nabiximols, the doses of CBD consumed in this product are very low (10-30 mg/day) and most likely inconsequential. ${ }^{43}$ In healthy volunteers, CBD had no clear analgesic effect in laboratory tests of pain thresholds and sensitivity, ${ }^{44}$ while a recent Australian clinical trial found that a single dose of adjunctive CBD (400 mg) was of no benefit in patients reporting to an emergency department with acute exacerbation of back pain..$^{45}$ However, a study of 20 patients with chronic neuropathic pain reported superiority of $120 \mathrm{mg} /$ day CBD over placebo. ${ }^{46} \mathrm{~A}$ recent observational study retrospectively assessed changes in quality of life in a subset of the first 400 New Zealand patients to receive prescription $\mathrm{CBD}$ (mostly $100 \mathrm{mg} \mathrm{CBD} / \mathrm{mL}$ oil administered by dropper) ${ }^{47}$ In this study, patients with non-cancer pain $(n=53)$ reported significant improvements in pain-related quality of life, improved mobility and reduced anxiety and depression. Surveys of users in countries where cannabis products are more freely available (eg North America) suggest that CBD-dominant products tend to be more frequently consumed for anxiety and depression, while THC-dominant products are preferentially used for pain and sleep. ${ }^{48}$ Current SAS-B data indicate that almost a quarter of current approvals for chronic pain involve Schedule 4 CBD-dominant products, despite the minimal evidence available regarding efficacy (Figure 4).

\section{Dosing and adverse effects}

A major challenge with the use of cannabinoids is to weigh up potential harms for patients versus clinical benefits. For a detailed examination of cannabis-related adverse effects and of driving-related issues, these authors recommend the articles of Arnold ${ }^{49}$ and Arkell et al, ${ }^{50}$ respectively.

\section{THC}

THC has well-documented side effects including dizziness, appetite stimulation, drowsiness, altered mood, anxiety, and impaired cognition and attention. These effects vary by dose and route of administration, and rapid tolerance can occur to such effects. In clinical trials, treatment-emergent side effects of typical oral THC doses (approximately 5-20 mg) tend to be mild or moderate in severity and more prominent on the first day of dosing. ${ }^{51,52}$ Patients using nabiximols generally report few adverse reactions, other than mild increases in appetite and some dizziness, nausea, fatigue and dysgeusia. Inhaled cannabis will produce more immediate and pronounced feelings of intoxication than oral THC products. ${ }^{53}$ In clinical practice, doses of THC should be slowly titrated upwards from $2.5-5 \mathrm{mg} /$ day to $10-20 \mathrm{mg} /$ day to avoid feelings of acute intoxication and other side effects (eg anxiety). Regular monitoring of patients for adverse effects is recommended. ${ }^{54}$
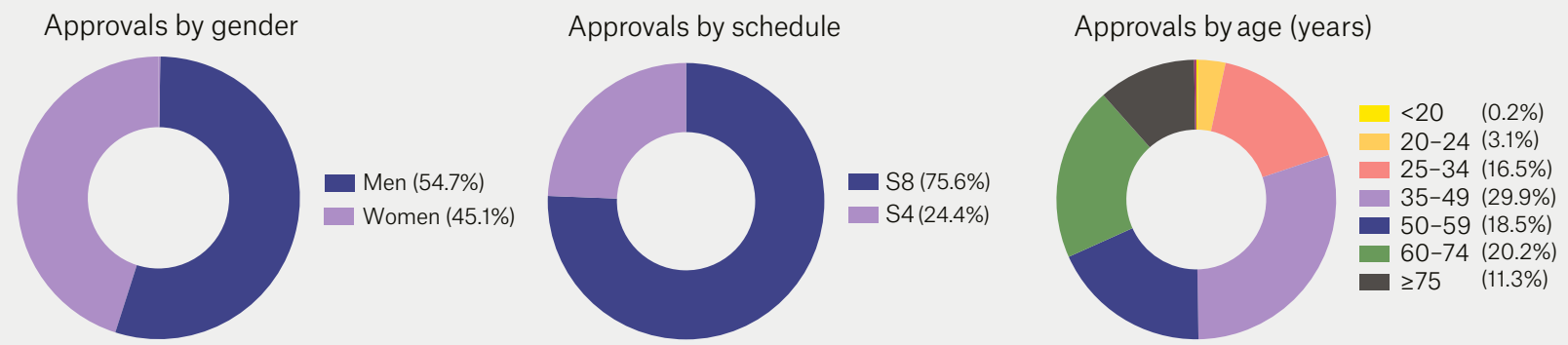

Figure 4. Other characteristics of Special Access Scheme Category B (SAS-B) approvals for chronic pain (month of April 2021)

A. There were more approvals for men than women (0.2\% did not specify gender); B. Approximately one-quarter of approvals were for cannabidiol-dominant (Schedule 4 [S4]) products, while the majority of approvals were for products containing substantial amounts of $\Delta^{9}$-tetrahydrocannabinol (Schedule 8 [S8]); C. Age breakdown of approvals showing very few approvals for patients under the age of 25 years. 
There is little evidence of tolerance to the analgesic effects of cannabisbased medicines during extended use. ${ }^{32}$ Unlike with opioids, hyperalgesia to painful stimuli does not appear to occur with chronic use of cannabis, ${ }^{55}$ and analgesic effects can be retained, even when tolerance to psychotropic effects have developed. ${ }^{56}$ Heavy cannabis use in vulnerable individuals can increase the risk of psychosis and schizophrenia, ${ }^{24,57}$ and THC is contraindicated in individuals with a family history of mental health problems. ${ }^{58}$ Caution is also advised when prescribing THC to patients under the age of 25 years, and SAS-B prescribing data show that very few approvals for chronic pain involve patients in this age group (Figure 4). The possibility of drug-seeking behaviour should be considered in otherwise healthy patients requesting a cannabis prescription. Adequate risk stratification for substance use disorder is suggested prior to initiating therapy. Additional cautions with THC products include active mood or anxiety disorder, heavy alcohol or opiate use, and pregnancy and breastfeeding. ${ }^{25,54}$

Legal prohibitions regarding THC and driving are a significant barrier to patient use: patients with a legitimate medicinal cannabis prescription are not exempt from current drug-driving laws. ${ }^{59}$ Dosing THC by night reduces side-effect burden and minimises the complications caused by daytime intoxication given that impairment has a maximal duration of approximately $8-10$ hours. ${ }^{60}$ Oral products are generally preferred to inhaled medicinal cannabis products because of issues related to respiratory health, although vaporised cannabis may allow more rapid relief for breakthrough pain, such as in cancer pain management.

\section{CBD}

CBD is well tolerated even at very high doses up to $6000 \mathrm{mg}$ and has relatively benign side effects, the most common being diarrhoea. ${ }^{61}$ Other side effects such as somnolence, decreased appetite and fatigue are mainly evident when other medications are co-administered. ${ }^{62}$ Clinical benefits of CBD are best seen at doses of 300-1500 mg in epilepsy, anxiety, psychosis and addictions. ${ }^{43,63}$ However, such high doses are expensive, so many patients and prescribers dose CBD at approximately $60-200 \mathrm{mg} /$ day. ${ }^{11,47}$ Prescribers should bear in mind the lack of evidence for efficacy of CBD at such low doses, although clinical trials using these dose ranges are underway.

CBD does not appear to impair driving and is not subject to current legal restrictions. ${ }^{59}$ Interactions between CBD and other prescription medications are possible given CBD inhibition of CYP450 enzymes. ${ }^{21}$ Interactions with the anticonvulsant clobazam are well documented in patients with epilepsy, ${ }^{64}$ and there are interactions with the commonly prescribed antidepressants citalopram and escitalopram that may increase their plasma concentrations. ${ }^{65}$ Upwards titration of CBD doses is therefore recommended as a precautionary principle, particularly in patients taking other medications.

\section{Other considerations regarding clinical use \\ Cannabinoids and opiates}

In addition to the effects on chronic pain itself, cannabinoids may reduce the requirement for patients to use conventional analgesics including opiates. ${ }^{66} \mathrm{CB} 1$ receptors and mu-opioid receptors are colocalised in pain processing brain regions, functionally interact ${ }^{67}$ and are involved in placebo analgesia. ${ }^{68}$ A recent study of 97 patients with two-year stable opioid use for chronic pain found that a CBD-rich gel allowed $50 \%$ of patients reduce their opioid medications, with two eliminating their need for opioids entirely. ${ }^{69}$

\section{Integrated approaches and benefits} The benefits of medicinal cannabis, in addition to potential pain reduction and opioid sparing, may include improved sleep, better quality of life and positive mood, all of which may contribute to improvements in chronic pain. ${ }^{70}$ This underscores the need to assess pain from a biopsychosocial perspective, including psychological, family, work and social influences. Management plans for chronic pain should include education, self-care strategies, behavioural management, multidisciplinary care, mindfulness, exercise and positive lifestyle. The use of cognitive behavioural therapy and stress management techniques is strongly recommended in addition to, or even instead of, pharmaceutical approaches or the use of medicinal cannabis. ${ }^{24,71}$

\section{Withdrawal from products}

Although medicinal cannabis is relatively safe when prescribed cautiously, patients with chronic pain who receive medicinal cannabis tend to be more likely to withdraw from clinical trials due to adverse effects than patients receiving placebo. ${ }^{24}$ Among people who use cannabis recreationally, abrupt discontinuation can produce a mild withdrawal syndrome characterised by sleep disturbances, depression and irritability, which typically peaks approximately two days following the last dose. ${ }^{72}$ Patients who have used THC products over several months or years are therefore advised to slowly taper off their use when withdrawing; inhaled cannabis can be replaced by oral products to facilitate dose titration during withdrawal. ${ }^{73} \mathrm{CBD}$ has no addiction or dependence liability, and sudden abstinence does not lead to withdrawal. ${ }^{74}$ Indeed, CBD is currently of significant interest as a potential therapeutic option in the treatment of addictions including drug withdrawal. ${ }^{75}$

\section{Conclusion}

Medicinal cannabis is worthy of consideration in the management of chronic pain, and it is important that doctors are aware of the positives and negatives related to its use. The more commonly prescribed oral products (oils, sprays and capsules) are attractive since they can be delivered in a more controlled and socially acceptable manner than inhaled products, although they have a slower onset. While CBD products are attractive given better safety when driving or performing other safety-sensitive tasks, current supportive evidence for their efficacy is limited. Harm minimisation should always be front of mind in prescribing decisions, particularly with 
patients who are driving regularly or using heavy machinery. It must be recognised that the long-term effects of medicinal cannabis, potential drug-drug interactions and efficacy across different pain types remain only partly understood. The guiding principle of start low, go slow is crucial, with the aim to obtain clinical benefits at the lowest possible dose and to minimise risks and side effects.

A recent and authoritative systematic review, commissioned by the International Association for the Study of Pain, concluded that the current evidence 'neither supports nor refutes claims of efficacy and safety for cannabinoids, cannabis, or cannabis-based medicines in the management of pain' and that there is 'the pressing need for studies to fill the research gap', ${ }^{76}$ a conclusion supported by another recent systematic review. ${ }^{23}$ The Faculty of Pain Medicine of the Australian and New Zealand College of Anaesthetists concluded that until higherquality evidence is available, currently available cannabinoid products should only be prescribed as part of a registered clinical trial. ${ }^{77}$

Despite the fact that current supportive evidence is of low overall quality, there are tens of thousands of patients with chronic pain being prescribed medicinal cannabis products in Australia, and hundreds of thousands using illicit cannabis products to self-medicate chronic pain. ${ }^{7,9}$ Many have legitimate lived experience of lasting pain reduction with cannabis that is not easily disregarded.,78,79 There is clearly a disconnection between the pronouncements of specialist medical colleges and current prescribing and community use of cannabinoids in Australia. It is hoped that the results of the next generation of clinical trials of cannabinoid products and pain will help to resolve this tension.

\section{Authors}

Luke A Henderson PhD, Professor, School of Medical Sciences (Neuroscience), University of Sydney, Sydney, NSW; Brain and Mind Centre, University of Sydney, Sydney, NSW

Vicki Kotsirilos AM, MBBS, FACNEM, FASLM, Honorary RACGP Fellowship, Adjunct Associate Professor, NICM Health Research Institute, Western Sydney University, Sydney, NSW
Elizabeth A Cairns PhD, Postdoctoral Research Associate, Lambert Initiative for Cannabinoid Therapeutics, Brain and Mind Centre, University of Sydney, Sydney, NSW; School of Psychology, Faculty of Science, University of Sydney, Sydney, NSW Alister Ramachandran FFPMANZCA, Anaesthetist and Pain Medicine Specialist, Pain Management Centre, Westmead Hospital, Westmead, NSW Chris C Peck PhD, Pain Management Specialist, Pain Management and Research Centre, Royal North Shore Hospital, St Leonards, NSW

lain S McGregor PhD, Academic Director, Lambert Initiative for Cannabinoid Therapeutics, Brain and Mind Centre, University of Sydney, Sydney, NSW; School of Psychology, Faculty of Science, University of Sydney, Sydney, NSW

Competing interests: ISM is an inventor on patents WO2018107216A1 and WO2017004674A1, licensed to Kinoxis Therapeutics involving use of novel small molecules (non-cannabinoid) to treat addictions and social deficits. ISM also has pending patents (PCT/AU2020/050941 and PCT/AU2019/903299) related to use of small molecules (non-cannabinoid) for treating weight gain and chronic pain, as well as patents WO2019227167 and WO2019071302 issued, which relate to cannabinoid therapeutics. ISM acts as an expert witness and consultant in legal cases involving the use of medical and non-medical cannabis.

Funding: EAC reports salary support from the Lambert Initiative for Cannabinoid Therapeutics, pledged by Barry and Joy Lambert to the University of Sydney. ISM reports research project funding from the National Health and Medical Research Council of Australia, and salary loading and research funding from the Lambert Initiative for Cannabinoid Therapeutics during the conduct of this study, as well as consulting fees from Kinoxis Therapeutics and an honorarium from Janssen Pharmaceuticals, outside the conduct of this study.

Provenance and peer review: Commissioned, externally peer reviewed.

Correspondence to:

luke.henderson@sydney.edu.au

\section{References}

1. Pain Australia. National strategic action plan for pain management. Deakin, ACT: Department of Health, 2019.

2. Deloitte Access Economics. The cost of pain in Australia. Canberra, ACT: Deloitte, 2019.

3. Raja SN, Carr DB, Cohen M, et al. The revised International Association for the Study of Pain definition of pain: Concepts, challenges, and compromises. Pain 2020;161(9):1976-82. doi: 10.1097/j.pain.0000000000001939.

4. Finnerup NB, Attal N, Haroutounian S, et al. Pharmacotherapy for neuropathic pain in adults: A systematic review and meta-analysis. Lancet Neurol 2015;14(2):162-73. doi: 10.1016/S14744422(14)70251-0.

5. Hylands-White N, Duarte RV, Raphael JH. An overview of treatment approaches for chronic pain management. Rheumatol Int 2017;37(1):29-42. doi: 10.1007/s00296-016-3481-8.

6. Australian Institute of Health and Welfare. National drug strategy household survey 2019. Canberra, ACT: AlHW, 2020.

7. Lintzeris N, Mills L, Suraev A, et al. Medical cannabis use in the Australian community following introduction of legal access: The 2018-2019 online cross-sectional Cannabis as Medicine Survey (CAMS-18). Harm Reduct J 2020;17(1):37. doi: 10.1186/s12954-020-00377-0.
8. Therapeutic Goods Administration. Freedom of information. Canberra, ACT: TGA, 2019.

9. Australian Institute of Health and Welfare. Emerging topic: Medicinal cannabis. Canberra, ACT: AlHW, 2019.

10. NPS MedicineWise. Medicinal cannabis: Process for prescribers. Surry Hills, NSW: NPS MedicineWise, 2020.

11. FreshLeaf Analytics. Australian medicinal cannabis market: Patient, product and pricing analysis. Sydney, NSW: FreshLeaf, 2021.

12. Chaput G, Med CP, Sussman J. Integrating primary care providers through the seasons of survivorship. Curr Oncol 2019;26(1):48-54. doi: 10.3747/co.26.4687.

13. Soliman N, Haroutounian S, Hohmann AG, et al. Systematic review and meta-analysis of cannabinoids, cannabis-based medicines, and endocannabinoid system modulators tested for antinociceptive effects in animal models of injury-related or pathological persistent pain. Pain 2021;162 Suppl 1:S26-44. doi: 10.1097/j. pain.0000000000002269.

14. Habib AM, Okorokov AL, Hill MN, et al. Microdeletion in a FAAH pseudogene identified in a patient with high anandamide concentrations and pain insensitivity. $\mathrm{Br} \mathrm{J}$ Anaesth 2019;123(2):e249-53. doi: 10.1016/j. bja.2019.02.019.

15. Cajanus K, Holmström EJ, Wessman M, Anttila V, Kaunisto MA, Kalso E. Effect of endocannabinoid degradation on pain: Role of FAAH polymorphisms in experimental and postoperative pain in women treated for breast cancer. Pain 2016;157(2):361-69.

16. Turcotte D, Doupe M, Torabi M, et al. Nabilone as an adjunctive to gabapentin for multiple sclerosis-induced neuropathic pain: A randomized controlled trial. Pain Med 2015 Jan;16(1):149-59. doi: 10.1111/pme.12569.

17. Bestard JA, Toth CC. An open-label comparison of nabilone and gabapentin as adjuvant therapy or monotherapy in the management of neuropathic pain in patients with peripheral neuropathy. Pain Pract 2011;11(4):353-68. doi: 10.1111/j.15332500.2010.00427.x.

18. Berlach DM, Shir Y, Ware MA. Experience with the synthetic cannabinoid nabilone in chronic noncancer pain. Pain Med 2006;7(1):25-29. doi: 10.1111/j.1526-4637.2006.00085.x.

19. Burstein S. Molecular mechanisms for the inflammation-resolving actions of lenabasum. Mol Pharmacol 2021;99(2):125-32. doi: 10.1124/ molpharm.120.000083.

20. Taylor L, Gidal B, Blakey G, Tayo B, Morrison G. A phase I, randomized, double-blind, placebocontrolled, single ascending dose, multiple dose, and food effect trial of the safety, tolerability and pharmacokinetics of highly purified cannabidiol in healthy subjects. CNS Drugs 2018;32(11):1053-67. doi: 10.1007/s40263-018-0578-5.

21. Balachandran P, Elsohly M, Hill KP. Cannabidiol interactions with medications, illicit substances, and alcohol: A comprehensive review. J Gen Intern Med 2021;36(7):2074-84. doi: 10.1007/s11606020-06504-8.

22. Moore RA, Fisher E, Finn DP, et al. Cannabinoids, cannabis, and cannabis-based medicines for pain management: An overview of systematic reviews. Pain 2021;162 Suppl 1:S67-79. doi: 10.1097/j. pain.0000000000001941.

23. Fisher E, Moore RA, Fogarty AE, et al. Cannabinoids, cannabis, and cannabis-based medicine for pain management: A systematic review of randomised controlled trials. Pain 
2021;162 Suppl 1:S45-66. doi: 10.1097/j. pain.0000000000001929.

24. Therapeutic Goods Administration. Guidance for the use of medicinal cannabis in the treatment of chronic non-cancer pain in Australia. Canberra, ACT: TGA, 2017.

25. Stockings E, Campbell G, Hall WD, et al. Cannabis and cannabinoids for the treatment of people with chronic noncancer pain conditions: A systematic review and meta-analysis of controlled and observational studies. Pain 2018;159(10):1932-54. doi: 10.1097/j.pain.0000000000001293.

26. Devon E. Vaporisers pass the TGA smoke screen. Retail Pharmacy Magazine. 13 September 2019.

27. Andreae $\mathrm{MH}$, Carter GM, Shaparin N, et al. Inhaled cannabis for chronic neuropathic pain: A meta-analysis of individual patient data. J Pain 2015;16(12):1221-32. doi: 10.1016/j. jpain.2015.07.009.

28. Wallace MS, Marcotte TD, Umlauf $A$, Gouaux B, Atkinson JH. Efficacy of inhaled cannabis on painful diabetic neuropathy. J Pain 2015;16(7):616-27. doi: 10.1016/j. jpain.2015.03.008.

29. Wilsey B, Marcotte T, Deutsch R, Gouaux B, Sakai S, Donaghe H. Low-dose vaporized cannabis significantly improves neuropathic pain. J Pain 2013;14(2):136-48. doi: 10.1016/j. jpain.2012.10.009.

30. Wilsey B, Marcotte TD, Deutsch R, Zhao H, Prasad $\mathrm{H}$, Phan A. An exploratory human laboratory experiment evaluating vaporized cannabis in the treatment of neuropathic pain from spinal cord injury and disease. J Pain 2016;17(9):982-1000. doi: 10.1016/j. jpain.2016.05.010.

31. Bar-Lev Schleider L, Mechoulam R, Lederman V, et al. Prospective analysis of safety and efficacy of medical cannabis in large unselected population of patients with cancer. Eur J Intern Med 2018;49:37-43. doi: 10.1016/j.ejim.2018.01.023.

32. Überall MA. A review of scientific evidence for THC: CBD oromucosal spray (nabiximols) in the management of chronic pain. J Pain Res 2020;13:399-410. doi: 10.2147/JPR.S240011.

33. Lichtman $A H$, Lux EA, McQuade R, et al. Results of a double-blind, randomized, placebo-controlled study of nabiximols oromucosal spray as an adjunctive therapy in advanced cancer patients with chronic uncontrolled pain. J Pain Symptom Manage 2018;55(2):179-88.e1. doi: 10.1016/j. jpainsymman.2017.09.001.

34. ClinicalTrials.gov. [Internet] Bethesda (MD): National Library of Medicine (US). 2012. Identifier NCT01606202, A study of cannabis based medicine extracts and placebo in patients with pain due to spinal cord injury. Available at https:// clinicaltrials.gov/ct2/show/results/NCT01606202. [Accessed 1 September 2021].

35. ClinicalTrials.gov. [Internet] Bethesda (MD): National Library of Medicine (US). 2008. Identifier NCT00710424, A double blind, randomized, placebo controlled, parallel group study of Sativex in the treatment of subjects with pain due to diabetic neuropathy. Available at https:// clinicaltrials.gov/ct2/show/NCT00710424 [Accessed 1 September 2021].

36. Lynch ME, Cesar-Rittenberg P, Hohmann AG A double-blind, placebo-controlled, crossover pilot trial with extension using an oral mucosal cannabinoid extract for treatment of chemotherapy-induced neuropathic pain. J Pain Symptom Manage 2014;47(1):166-73. doi: 10.1016/j.jpainsymman.2013.02.018.

37. Berman JS, Symonds C, Birch R. Efficacy of two cannabis based medicinal extracts for relief of central neuropathic pain from brachial plexus avulsion: Results of a randomised controlled trial. Pain 2004;112(3):299-306. doi: 10.1016/j. pain.2004.09.013.

38. Nurmikko TJ, Serpell MG, Hoggart B, Toomey PJ, Morlion BJ, Haines D. Sativex successfully treats neuropathic pain characterised by allodynia: A randomised, double-blind, placebo-controlled clinical trial. Pain 2007;133(1-3):210-20. doi: 10.1016/j.pain.2007.08.028.

39. Ueberall MA, Essner U, Mueller-Schwefe GH Effectiveness and tolerability of THC:CBD oromucosal spray as add-on measure in patients with severe chronic pain: Analysis of 12-week open-label real-world data provided by the German Pain e-Registry. J Pain Res 2019;12:1577-604. doi: 10.2147/JPR.S192174.

40. Iskedjian M, Bereza B, Gordon A, Piwko C, Einarson TR. Meta-analysis of cannabis based treatments for neuropathic and multiple sclerosisrelated pain. Curr Med Res Opin 2007;23(1):17-24. doi: 10.1185/030079906x158066.

41. Svendsen KB, Jensen TS, Bach FW. Does the cannabinoid dronabinol reduce central pain in multiple sclerosis? Randomised double blind placebo controlled crossover trial. BMJ 2004;329(7460):253. doi: 10.1136/ bmj.38149.566979.AE

42. Rintala DH, Fiess RN, Tan G, Holmes SA, Bruel BM. Effect of dronabinol on central neuropathic pain after spinal cord injury: A pilot study. Am J Phys Med Rehabil 2010;89(10):840-48 doi: 10.1097/PHM.0b013e3181f1c4ec.

43. Millar SA, Stone NL, Bellman ZD, Yates AS, England TJ, O'Sullivan SE. A systematic review of cannabidiol dosing in clinical populations. $\mathrm{Br} J$ Clin Pharmacol 2019;85(9):1888-900. doi: 10.1111/ bcp.14038.

44. Arout CA, Haney M, Herrmann ES, Bedi G, Cooper ZD. A placebo-controlled investigation of the analgesic effects, abuse liability, safety and tolerability of a range of oral cannabidiol doses in healthy humans. Br J Clin Pharmacol 2021. doi: 10.1111/bcp.14973. Epub ahead of print.

45. Bebee B, Taylor DM, Bourke E, et al. The CANBACK trial: A randomised, controlled clinical trial of oral cannabidiol for people presenting to the emergency department with acute low back pain. Med J Aust 2021;214(8):370-75. doi: 10.5694/ mja2.51014.

46. Wade DT, Robson P, House H, Makela P, Aram J. A preliminary controlled study to determine whether whole-plant cannabis extracts can improve intractable neurogenic symptoms. Clin Rehabil 2003;17(1):21-29.

47. Gulbransen G, Xu W, Arroll B. Cannabidiol prescription in clinical practice: An audit on the first 400 patients in New Zealand. BJGP Open 2020;4(1):bjgpopen20X101010. doi: 10.3399/ bjgpopen20X101010.

48. Kalaba M, Ware MA. Cannabinoid profiles in medical cannabis users: Effects of age, gender, symptoms, and duration of use. Cannabis Cannabinoid Res 2021. doi: 10.1089/ can.2020.0120. Epub ahead of print.

49. Arnold JC. A primer on medicinal cannabis safety and potential adverse effects. Aust J Gen Pract 2021;50(6):345-50. doi: 10.31128/AJGP-02-21-5845.

50. Arkell TR, Vinckenbosch F, Kevin RC, Theunissen EL, McGregor IS, Ramaekers JG. Effect of cannabidiol and $\Delta 9$-tetrahydrocannabinol on driving performance: $A$ randomized clinical trial. JAMA 2020;324(21):2177-86. doi: 10.1001/ jama.2020.21218.

51. Peters EN, Mosesova I, MacNair L, et al. Safety, pharmacokinetics, and pharmacodynamics of spectrum red softgels in healthy participants. J Anal Toxicol 2021:bkab035. doi: 10.1093/jat/ bkab035. Epub ahead of print.

52. Peters EN, Mosesova I, MacNair L, et al. Safety, pharmacokinetics, and pharmacodynamics of spectrum yellow oil in healthy participants. J Anal Toxicol 2021:bkab026. doi: 10.1093/jat/bkab026. Epub ahead of print.

53. Spindle TR, Martin EL, Grabenauer M, Woodward T, Milburn MA, Vandrey R. Assessment of cognitive and psychomotor impairment, subjective effects, and blood $\mathrm{THC}$ concentrations following acute administration of oral and vaporized cannabis. J Psychopharmacol 2021;35(7):786-803. doi: $10.1177 / 02698811211021583$.

54. Therapeutic Goods Administration. Guidance for the use of medicinal cannabis in Australia: Overview. Version 1. Canberra, ACT: TGA, 2017.

55. St Pierre M, Russo EB, Walsh Z. No evidence of altered reactivity to experimentally induced pain among regular cannabis users. Clin J Pain 2020;36(8):589-93. doi: 10.1097/ AJP.0000000000000844.

56. Pertwee RG. Emerging strategies for exploiting cannabinoid receptor agonists as medicines. $\mathrm{Br} J$ Pharmacol 2009;156(3):397-411. doi: 10.1111/j.14765381.2008.00048.x

57. National Academies of Sciences, Engineering, and Medicine; Health and Medicine Division; Board on Population Health and Public Health Practice; Committee on the Health Effects of Marijuana: An Evidence Review and Research Agenda. The health effects of cannabis and cannabinoids: The current state of evidence and recommendations for research. Washington, DC: National Academies Press, 2017

58. Bourque J, Afzali MH, Conrod PJ. Association of cannabis use with adolescent psychotic symptoms. JAMA Psychiatry 2018;75(8):864-66. doi: 10.1001/jamapsychiatry.2018.1330.

59. Arkell TR, McCartney D, McGregor IS. Medical cannabis and driving. Aust J Gen Pract 2021;50(6):357-62. doi: 10.31128/AJGP-02-21-5840.

60. McCartney D, Arkell TR, Irwin C, McGregor IS. Determining the magnitude and duration of acute $\Delta 9$-tetrahydrocannabinol ( $\triangle 9$-THC)induced driving and cognitive impairment: $A$ systematic and meta-analytic review. Neurosc Biobehav Rev 2021;126:175-93. doi: 10.1016/j. neubiorev.2021.01.003.

61. Chesney E, Oliver D, Green A, et al. Adverse effects of cannabidiol: A systematic review and meta-analysis of randomized clinical trials. Neuropsychopharmacology 2020;45(11):1799-806 doi: 10.1038/s41386-020-0667-2.

62. Iffland K, Grotenhermen F. An update on safety and side effects of cannabidiol: A review of clinical data and relevant animal studies. Cannabis Cannabinoid Res 2017;2(1):139-54. doi: 10.1089/ can.2016.0034.

63. McGregor IS, Cairns EA, Abelev S, et al. Access to cannabidiol without a prescription: A cross-country comparison and analysis. Int J Drug Policy 2020;85:102935. doi: 10.1016/j. drugpo.2020.102935.

64. VanLandingham KE, Crockett J, Taylor L, Morrison G. A phase 2, double-blind, placebocontrolled trial to investigate potential drug-drug interactions between cannabidiol and clobazam. J Clin Pharmacol 2020;60(10):1304-13. doi: 10.1002/jcph.1634.

65. Anderson LL, Doohan PT, Oldfield L, et al. Citalopram and cannabidiol: In vitro and in vivo evidence of pharmacokinetic interactions relevant to the treatment of anxiety disorders in young 
people. J Clin Psychopharmacol 2021. doi: 10.1097/ JCP.0000000000001427. Epub ahead of print.

66. Boehnke KF, Litinas E, Clauw DJ. Medical cannabis use is associated with decreased opiate medication use in a retrospective cross-sectional survey of patients with chronic pain. J Pain 2016;17(6):739-44. doi: 10.1016/j. jpain.2016.03.002.

67. Salio C, Fischer J, Franzoni MF, Mackie K, Kaneko T, Conrath M. CB1-cannabinoid and mu-opioid receptor co-localization on postsynaptic target in the rat dorsal horn. Neuroreport 2001;12(17):3689-92. doi: 10.1097/00001756200112040-00017.

68. Peciña M, Martínez-Jauand M, Hodgkinson C, Stohler CS, Goldman D, Zubieta JK. FAAH selectively influences placebo effects. Mol Psychiatry 2014;19(3):385-91. doi: 10.1038/ mp.2013.124.

69. Capano A, Weaver R, Burkman E. Evaluation of the effects of CBD hemp extract on opioid use and quality of life indicators in chronic pain patients: A prospective cohor study. Postgrad Med 2020;132(1):56-61. doi: 10.1080/00325481.2019.1685298.

70. Hulland O, Oswald J. Cannabinoids and pain: The highs and lows. Rheum Dis Clin North Am 2021;47(2):265-75. doi: 10.1016/j.rdc.2020.12.005.

71. Wan A. GP pain management: What are the 'Ps' and 'As' of pain management? Aust Fam Physician 2014;43(8):537-40.

72. Bonnet U, Preuss UW. The cannabis withdrawal syndrome: Current insights. Subst Abuse Rehabil 2017;8:9-37. doi: 10.2147/SAR.S109576.

73. Lintzeris N, Bhardwaj A, Mills L, et al. Nabiximols for the treatment of cannabis dependence: A randomized clinical trial. JAMA Intern Med 2019;179(9):1242-53. doi: 10.1001/ jamainternmed.2019.1993.

74. Taylor L, Crockett J, Tayo B, Checketts D, Sommerville K. Abrupt withdrawal of cannabidiol (CBD): A randomized trial. Epilepsy Behav 2020;104 Pt A:106938. doi: 10.1016/j. yebeh.2020.106938.
75. Chye Y, Christensen E, Solowij N, Yücel M. The endocannabinoid system and cannabidiol's promise for the treatment of substance use disorder. Front Psychiatry 2019;10:63. doi: 10.3389/fpsyt.2019.00063.

76. Haroutounian S, Arendt-Nielsen L, Belton J, et al. IASP Presidential Task Force on Cannabis and Cannabinoid Analgesia: Research agenda on the use of cannabinoids, cannabis, and cannabis-based medicines for pain management. Pain 2021;162 Suppl 1:S117-24. doi: 10.1097/j. pain.0000000000002266.

77. Australian and New Zealand College of Anaesthetists Faculty of Pain Medicine. FPM and medicinal cannabis. Melbourne, Vic: ANZCA, 2021. Available at www.anzca.edu.au/news/topnews/fpm-and-medicinal-cannabis [Accessed 5 September 2021].

78. Perkins D, Brophy H, McGregor IS, et al. Medicinal cannabis and driving: The intersection of health and road safety policy. Int J Drug Policy 2021;97:103307. doi: 10.1016/j.drugpo.2021.103307. Epub ahead of print.

79. Duns G. Editorial: Medical cannabis - Progress and promise. Aust J Gen Pract 2021;50(6):341. doi: 10.31128/AJGP-06-21-1234e. 